OPEN ACCESS

Edited by:

Rifat Hamoudi,

University of Sharjah,

United Arab Emirates

Reviewed by:

Min Li,

University of Oklahoma Health

Sciences Center, United States

Jingxuan Yang,

University of Texas Health Science Center at Houston, United States

Rachel Zhang,

Weill Cornell Medical Center,

United States

*Correspondence:

Man Hu

human5770@163.com

Jin-Ming Yu

sdyujinming@163.com

Specialty section:

This article was submitted to

Cancer Immunity

and Immunotherapy,

a section of the journal

Frontiers in Immunology

Received: 05 July 2020 Accepted: 08 October 2020 Published: 30 October 2020

Citation:

Ding X-C, Wang L-L, Zhu Y-F, Li Y-D,

Nie S-L, Yang J, Liang $H$,

Weichselbaum RR, Yu J-M and Hu M

(2020) The Change of Soluble

Programmed Cell Death-Ligand 1 in

Glioma Patients Receiving

Radiotherapy and Its Impact on

Clinical Outcomes.

Front. Immunol. 11:580335.

doi: 10.3389/fimmu.2020.580335

\section{The Change of Soluble Programmed Cell Death-Ligand 1 in Glioma Patients Receiving Radiotherapy and Its Impact on Clinical Outcomes}

\author{
Xing-Chen Ding ${ }^{1,2}$, Liang-Liang Wang ${ }^{3}$, Yu-Fang Zhu ${ }^{2}$, Yan-Dong $\mathrm{Li}^{4}$, Shu-Lun $\mathrm{Nie}^{1,2}$, \\ Jia Yang ${ }^{2}$, Hua Liang ${ }^{3}$, Ralph R. Weichselbaum ${ }^{3}$, Jin-Ming Yu ${ }^{2 *}$ and $\mathrm{Man} \mathrm{Hu}^{2 *}$ \\ 1 Department of Oncology, Shandong First Medical University and Shandong Academy of Medical Sciences, Jinan, China, \\ 2 Department of Radiation Oncology, Shandong Cancer Hospital and Institute, Shandong First Medical University and \\ Shandong Academy of Medical Sciences, Jinan, China, ${ }^{3}$ Department of Radiation and Cellular Oncology, Ludwig Center for \\ Metastasis Research, The University of Chicago, Chicago, IL, United States, ${ }^{4}$ Department of Anesthesiology, Affiliated \\ Hospital of Jining Medical University, Jinan, China
}

Background: The programmed cell death ligand 1 (PD-L1) plays a key role in glioma development. However, due to the specificity of glioma's anatomical position, the role of its expression as a tumor biomarker is limited. It has been proven that the levels of soluble programmed death-ligand 1 (SPD-L1) are associated with prognosis in many malignancies including glioma. However, the expression of SPD-L1 in glioma patients receiving radiotherapy $(R T)$ remains unclear. The purpose of this study was to evaluate the concentration of SPD-L1 in the plasma of glioma patients before and after RT and to explore its relationship with clinical outcomes.

Methods: Between October 2017 and September 2018, glioma patients treated with RT (30 \pm 10 Gy, 2 Gy/f) were enrolled, and blood samples were collected before and after RT. We quantified the SPD-L1 levels by enzyme-linked immunosorbent assay (ELISA). The isocitrate dehydrogenase-1 (IDH-1) mutational status and Ki-67 expression of tumors were evaluated by immunohistochemistry. Glioma murine model were used to address whether circulating SPD-L1 molecules are directly targeted by an anti-PD-L1 antibody. The associations between SPD-L1 and clinical features were assessed with Pearson's or Spearman's correlation analysis. The progression-free survival (PFS) and overall survival (OS) were determined by the Kaplan-Meier method.

Results: Sixty glioma patients were included, with a median age of 52 years. The proportions of grade I, II, III, and IV gliomas were $6.7 \%, 23.3 \%, 28.4 \%$, and $41.6 \%$, respectively. The baseline SPD-L1 levels were significantly associated with tumor grade, IDH-1 mutation status and Ki-67 expression. Using $14.35 \mathrm{pg} / \mathrm{ml}$ as the cutoff, significantly worse PFS and OS were both observed in patients with higher baseline levels of SPD-L1 $(P=0.027$ and 0.008 , respectively). RT significantly increased the mean level of SPD-L1 $(P<0.001)$. Further analysis showed that the level of SPD-L1 in IDH-1 mutation patients was higher than that in wild-type 
patients. Furthermore, an analysis of glioma murine model indicated that anti-PD-L1 antibody combine with $\mathrm{RT}$ can be a potentially powerful cancer therapy.

Conclusion: This study reported that SPD-L1 might be a potential biomarker to predict the outcome in glioma patients receiving RT. The elevated level of SPD-L1 after RT suggested that the strategy of a combination of immune checkpoint inhibitors and RT might be promising for glioma patients, especially for those with $\mathrm{IDH}-1$ mutations.

Keywords: soluble programmed cell death-ligand 1, glioma, radiotherapy, clinical significance, prognosis

\section{INTRODUCTION}

Gliomas are the most common primary brain tumors, with a 5year overall survival (OS) of approximately $36 \%$ (1). Glioblastoma (GBM) with only $5.6 \%$ of 5 -year OS, is the most aggressive form making up 54\% of all gliomas (1). Despite neurosurgical resection and adjuvant radio- and chemotherapy prolonging patient survival times, most glioma patients relapse and have limited their life expectancy. The reasons for the failure of conventional therapies include the protection of tumor cells by the blood-brain barrier, as well as invasive tumor growth in an essential organ, which limits the utility of local therapy (2). It is anticipated that novel effective approaches will be urgently required for the systemic treatments of gliomas.

Anti- programmed death 1/programmed death ligand 1 (PD1/PD-L1) immunotherapy has shown clinical efficacy against many different solid tumor types and hematological malignancies (3-6). However, clinical trials of anti-PD-1/PDL1 immunotherapy for glioma are relatively delayed $(7,8)$. Only one phase III clinical trial, Checkmate 143, has been completed; however, nivolumab did not exhibit increased survival benefits compared with bevacizumab (9). It seems that the PD-1/PD-L1 axis only plays one role in the malignant biological behavior of gliomas, while other molecular signaling networks may also play indispensable roles. Radiation is commonly used to treat glioma patients and has been identified to activate immune responses (10-12). Thus, researchers tried to explore the clinical efficacy of immunotherapy (nivolumab) + radiotherapy (RT) \pm temozolomide (TMZ) in newly diagnosed glioblastoma patients in some ongoing phase III clinical trials, including Checkmate 498 (NCT02617589) and Checkmate 548 (NCT02667587). However, challenges remain to be addressed to maximize the efficacy of this promising combination. One of these challenges is the identification of biomarkers to assess the dynamic changes in the immune system at the level of patients undergoing RT.

To date, a number of candidate biomarkers of the immune response during and after $\mathrm{RT}$, including both circulating and cellular, have been reported (13-17). Increasing evidence suggests that the expression of soluble PD-L1 (sPD-L1) in the blood is significantly associated with prognosis in gliomas and several extracranial malignancies (18-20). The elevated circulating and cerebrospinal fluid sPD-L1 levels were associated with aggressive biological activities in glioma patients (21). Similarly, high sPD-L1 levels were found which were correlated with abdominal organ metastasis in patients with advanced non-small cell lung cancer (NSCLC) (18) and increased mortality risk in patients with hepatocellular carcinoma (HCC) (20). Moreover, both sPD-1 and SPD-L1 could also acted as useful biomarkers to predict the outcome of PD-1 inhibition therapy in melanoma patients (22). These preliminary results prompted us to further investigate the application of sPD-L1 in the treatment of tumors.

Given the limited evidence that SPD-L1 may be a biomarker to predict the response to immunotherapy in gliomas, our study was performed to evaluate plasma concentrations of sPD-L1 before and after RT in glioma patients and to investigate the relationship of sPD-L1 levels with clinical outcomes. We hypothesized that circulating sPD-L1 molecules in the blood would deliver systemic inhibitory messages that could globally adversely impact antitumoral immune responses.

\section{MATERIALS AND METHODS}

\section{Patients}

In this study, 60 patients were recruited. All of them were diagnosed primary or recurrent glioma, then treated with RT for glioma in Shandong Cancer Hospital between October 2017 and September 2018. The study protocol was approved by the Ethics Committee of the Shandong Cancer Hospital, and all patients gave their written informed consent prior to study inclusion. The optimal treatment option was determined by a multidisciplinary tumor board in accordance with our institution's treatment policy. RT was performed using conventional fractionated RT. It was considered in patients on an individual basis, with a total dose of 54-60 Gy in 30 fractions (f). Concurrent peroral chemotherapy with TMZ was administered at $75 \mathrm{mg} / \mathrm{m}^{2}$ daily during RT. Bevacizumab combined with CCRT was administered at $10 \mathrm{mg} / \mathrm{kg}$ (repeated every 2 weeks). If the patient was required surgery first, RT was initiated within 8 weeks after surgery. After the scheduled treatment was finished, regular follow-up was conducted every 3 months with imaging studies and tumor marker analyses.

\section{Blood Sampling}

Blood (5-10 ml) was collected from the patients before RT (0 Gy) and after RT (30 $\pm 10 \mathrm{~Gy}, 2 \mathrm{~Gy} / \mathrm{f})$ using aseptic tubes containing EDTA (5 ml). The blood samples were centrifuged at $2000 \mathrm{rpm}$ for $10 \mathrm{~min}$ at $4^{\circ} \mathrm{C}$ to separate the plasma. Additional centrifugation for $10 \mathrm{~min}$ was performed to produce cell-free plasma, after which the 
plasma aliquots were immediately frozen at $-80^{\circ} \mathrm{C}$ for further analysis.

\section{Soluble PD-L1 Measurement}

Patients' sPD-L1 levels were measured using the human PD-L1 simple-step enzyme-linked immunosorbent assay kit (ab214565, Abcam, USA). In brief, $50 \mu$ l of standards at different concentrations and patient plasma samples were added to the wells. Subsequently, $50 \mu \mathrm{l}$ of PD-L1-conjugated antibody was added, incubated for $1 \mathrm{~h}$ at room temperature and then washed 3 times. Next, the substrate solution was applied for the color reaction, which was stopped with stop solution, and the absorbance was immediately measured at $450 \mathrm{~nm}$ using an enzyme-linked immunosorbent assay reader (VERSA max microplate reader; USA). The sPD-L1 level was calculated according to standard curves. The minimum detectable concentration of sPD-L1 was $2.91 \mathrm{pg} / \mathrm{ml}$.

\section{Immunohistochemistry and Molecular Pathology}

The immunohistochemistry (IHC) sample slides were reviewed by two neuropathologists, and a systematic neuropathological review was based on the 2007 WHO classification of CNS tumors. Tumor tissue was formalin fixed and paraffin embedded according to standard laboratory practice. Specimens were stained with antibodies against isocitrate dehydrogenase-1 (IDH-1) R132H (clone H09, 1:50 dilution; Maxim, China) and Ki-67 (MIB1; Santa Cruz, Shanghai, China, 1:50 dilution). Cells with pale brown granular deposits were considered to have IDH-1 mutational status and be Ki-67 positive. The Ki-67 index is the percentage of positive cells in the densest visual field. IHC analyses were then performed with a quantitative approach under a light microscope.

\section{Glioma Murine Model}

C57BL/6 mice (6-8 weeks) were maintained in SPF laboratory conditions and were subcutaneously injected with $2 \times 10^{6}$ cells (GL261 cells) in the right flank (day 0). When the tumor reached a volume of approximately $100 \mathrm{~mm}^{3}$ (approximately 10 days), tumors received one dose of $20 \mathrm{~Gy}$ ionizing radiation (IR), and/or $200 \mu \mathrm{g}$ of anti-PD-L1 antibody (clone 10F.9G2) or isotype control antibody. The sPD-L1 level in the plasma was measured using the mouse PD-L1 DuoSet ELISA (DY1019-05, R\&D Systems, USA) before and after IR. Tumor volume was measured twice weekly with calipers, and tumor volume was approximated using the equation for an ellipsoid: (width) ${ }^{2} \times$ length/2. Mice were sacrificed when tumors reached $2,000 \mathrm{~mm}^{3}$.

For the suppression assay, $\mathrm{CD}^{+} \mathrm{T}$ cells were purified from mouse lymph nodes (inguinal, axillary, brachial, superficial cervical, and lumbar) and spleens by CD8 isolation kit (Stemcell, Vancouver, BC). $2 \times 10^{5}$ of naïve $\mathrm{CD}^{+} \mathrm{T}$ cells and the plasma of mice after IR were co-cultured in complete RPMI1640 with the presence of $100 \mu \mathrm{M}$ of $\beta$-mercapitoethanol (Sigma) and $2 \mu \mathrm{g} / \mathrm{ml} \alpha \mathrm{CD} 28$ (Biolegend, clone 37.51) in the wells of a flat-bottom 96-well plate coated with $5 \mu \mathrm{g} / \mathrm{ml} \alpha \mathrm{CD} 3$ (Biolegend, clone 145-2C11). Cells were harvested after 3 days, stained for $\mathrm{CD}^{+} \mathrm{T}$ cells (Biolegend, clone 53-6.7) and analyzed by flow cytometry.

All experiments related to animals were strictly performed in accordance with guidelines approved by the Ethics Committee of Shandong Cancer Hospital.

\section{Statistical Analysis}

Continuous variables are shown as the mean \pm the standard deviation (SD) and the minimum-maximum range. The differences between the two groups were calculated using the t-test or the Mann-Whitney U-test according to the normality of the data. The Kruskal-Wallis test was used for the comparison of three or more groups. The post hoc Bonferroni test was used for multiple comparisons. Correlations between the sPD-L1 level and clinical factors were analyzed using Pearson's correlation analysis or Spearman's correlation analysis for continuous variables. The chi-squared test or Fisher's exact test were used for categorical variables. Receiver operating characteristic (ROC) curve analysis was used to determine the optimal cut-off value of sPD-L1 and Ki-67 expression rates. The survival duration was calculated from the date of disease diagnosis (RT start) to the corresponding event. The Kaplan-Meier method with the log-rank test was used to compare survival between groups. Multivariable analysis was carried out by the Cox regression hazard model. The dynamics of sPD-L1 in the plasma were analyzed by the mixed-model approach. All statistical tests were two-sided, and $P$ values $<0.05$ were considered to be significant. All data were analyzed using IBM SPSS software version 22.0 (IBM, New York, USA). Figures were made by GraphPad Prism version 5.00 (San Diego, California, USA).

\section{RESULTS}

\section{Patient Characteristics and Survival Outcome}

In this study, 60 glioma patients who had measurable tumors and received RT in Shandong Cancer Hospital were enrolled. Of them, 33 were female and 27 were male, with a median age of 52 years (range, 18-75). Fifty-two out of 60 patients received a pathological diagnosis (20 via subtotal resections and 32 via tumor biopsies), and the other eight patients were diagnosed with GBM by radiological findings based on the current guidelines. Twenty-five patients (41.6\%) had pathological grade IV gliomas, 17 patients (28.4\%) had grade III gliomas, 14 patients $(23.3 \%)$ had grade II gliomas, and four patients $(6.7 \%)$ had grade I gliomas. Of the 60 patients, $42(70 \%)$ patients received RT plus TMZ (CRT), 10 (16.7\%) patients received RT plus both TMZ and bevacizumab (CRT+T), and the other eight patients received only RT. The clinical baseline characteristics and outcomes of 60 glioma patients were systematically reviewed, and the results are summarized in Table $\mathbf{1}$. 
TABLE 1 | Patient characteristics and outcomes.

\begin{tabular}{|c|c|}
\hline Parameter & Patients \\
\hline \multicolumn{2}{|l|}{ Epidemiology } \\
\hline Patients, $n$ & 60 \\
\hline Gender, m/f (\%) & $27 / 33(45 / 55)$ \\
\hline Age, median, range & $52,18-75$ \\
\hline Karnofsky performance score, median, range & $90,60-90$ \\
\hline Numerical Rating Scale (pain measurement), median, range & $0,0-6$ \\
\hline Nutritional Risk Screening 2002, median, range & $1,0-3$ \\
\hline \multicolumn{2}{|l|}{ Pathological grading } \\
\hline $\ln (\%)$ & $4(6.7)$ \\
\hline II n (\%) & $14(23.3)$ \\
\hline III n (\%) & $17(28.4)$ \\
\hline IV n (\%) & $25(41.6)$ \\
\hline \multicolumn{2}{|l|}{ Tumor position } \\
\hline Left brain, n (\%) & $27(45)$ \\
\hline Right brain, n (\%) & $11(18.3)$ \\
\hline Brain stem, n (\%) & $9(15)$ \\
\hline Other, n (\%) & $13(21.7)$ \\
\hline \multicolumn{2}{|l|}{ IDH-1 status } \\
\hline Mutation, n (\%) & $15(25)$ \\
\hline Wild type, n (\%) & $25(41.7)$ \\
\hline Unknown, n (\%) & $20(33.3)$ \\
\hline \multicolumn{2}{|l|}{ Ki-67 expression (cut off rate) } \\
\hline$\leq 27.5 \%, \mathrm{n}(\%)$ & $28(46.6)$ \\
\hline$>27.5 \%, \mathrm{n}(\%)$ & $16(26.7)$ \\
\hline Unknown, n (\%) & $16(26.7)$ \\
\hline \multicolumn{2}{|l|}{ Diagnostic style } \\
\hline Subtotal resection, n (\%) & 20 (33.3) \\
\hline Tumor biopsy, n (\%) & $32(53.4)$ \\
\hline No biopsy, n (\%) & $8(13.3)$ \\
\hline \multicolumn{2}{|l|}{ Type of therapy } \\
\hline Radiotherapy + TMZ, n (\%) & $42(70)$ \\
\hline Radiotherapy + TMZ + bevacizumab, n (\%) & $10(16.7)$ \\
\hline Radiotherapy only, n (\%) & $8(13.3)$ \\
\hline Follow-up time, median, range & $28.7(5.4-38.7)$ \\
\hline \multicolumn{2}{|l|}{ Recurrence and/or metastasis at last follow-up } \\
\hline Yes, n (\%) & $23(38.3)$ \\
\hline No, n (\%) & $28(46.7)$ \\
\hline Unknown, n (\%) & $9(15)$ \\
\hline \multicolumn{2}{|l|}{ Alive at last follow-up } \\
\hline Yes, n (\%) & 38 (63.3) \\
\hline No, n (\%) & $22(36.7)$ \\
\hline Unknown, n (\%) & $0(0)$ \\
\hline
\end{tabular}

\section{Association Between Baseline sPD-L1 Levels and Clinical Factors}

To investigate the association between baseline sPD-L1 and clinical factors, we measured the sPD-L1 levels in the plasma of 60 patients before radiation therapy and detected the IDH-1 mutational status of 40 patients and the expression of Ki-67 in 44 patients by IHC. The mean level of baseline sPD-L1 was $47.39 \pm$ $59.01 \mathrm{pg} / \mathrm{ml}$ (range, $0-283.13 \mathrm{pg} / \mathrm{ml}$ ). Spearman correlation analysis showed that the baseline sPD-L1 level was positively associated with tumor grade $(r=0.495, P<0.001)$, IDH-1 mutational status $(r=0.379, P=0.016)$, and Ki-67 expression rate $(r=0.434, P=0.003)$. With the increase in glioma stage, the mean level of baseline sPD-L1 tended to increase (stage I: $8.18 \pm$ $2.70 \mathrm{pg} / \mathrm{ml}$; stage II: $10.52 \pm 18.35 \mathrm{pg} / \mathrm{ml}$; stage III: $29.65 \pm 24.23$ $\mathrm{pg} / \mathrm{ml}$, and stage IV: $60.60 \pm 65.95 \mathrm{pg} / \mathrm{ml}$, Figure 1A). Compared to patients with IDH-1 wild-type (WT) tumors, patients with
IDH-1 mutation (MUT) tumors showed markedly lower levels of baseline sPD-L1 in plasma $(17.28 \pm 24.59 \mathrm{pg} / \mathrm{ml} v s .61 .18 \pm 64.30$ $\mathrm{pg} / \mathrm{ml}$, Figure 1B). In addition, we found that the sPD-L1 level was higher in patients with $\mathrm{Ki}-67>27.5 \%$ than in those with Ki$67 \leq 27.5 \%(82.58 \pm 70.77 \mathrm{pg} / \mathrm{ml} v s .24 .68 \pm 27.89 \mathrm{pg} / \mathrm{ml}$, Figure 1C). As expected, there were no significant associations between sPD-L1 levels and other factors, e.g., sex, age, Karnofsky Performance Status (KPS) score, Numerical Rating Scale (NRS), Nutritional Risk Screening 2002 (NRS 2002), or tumor location.

\section{Correlation Between Baseline sPD-L1 Levels and Clinical Outcomes}

The median follow-up duration was 28.7 (range, 5.4-38.7) months. The disease of $23 / 60$ (38.3\%) patients progressed, and $22 / 60(36.7 \%)$ patients died within the observation time. The median OS and progression-free survival (PFS) were 28.7 months and 23.2 months, respectively. To evaluate the predictive value of the baseline sPD-L1 level for survival, a cutoff value of $14.35 \mathrm{pg} / \mathrm{ml}$ was obtained using ROC curve analysis (AUC $=0.73 ; P=0.003)$. Thirty-three patients $(55 \%)$ had high sPD-L1 levels (> 14.35 pg/ml), and the other 27 patients had low sPD-L1 levels $(\leq 14.35 \mathrm{pg} / \mathrm{ml})$. Significantly worse median OS was noted in patients with higher baseline SPD-L1 levels than in those with lower baseline sPD-L1 levels (23.1 vs. 28.7 months, $P=0.008$; Figure 2A). Additionally, patients with decreased sPD-L1 after RT had significantly worse median OS (20.8vs. 29.5 months, $P=0.040)$ (Figure 2B). Other factors, including IDH-1 WT tumors $(P=0.036)$, GBMs $(P=0.010)$, tumors located in the brainstem $(P=0.001)$ and tumors with a Ki-67 expression rate $>27.5 \%(P=0.001)$, can also affect the OS of patients (Figures 2C-F). In this study, changes in sPD-L1 levels, IDH-1 mutational status and tumor location were independent prognostic factors $(P=0.003, \mathrm{HR}=0.019,95 \%$ CI: $0.001-$ $0.268 ; P=0.011$, HR $=0.029,95 \%$ CI: $0.002-0.448 ; P=0.002$, HR $=26.302$, 95\% CI: 3.239-213.550) (Table 2). However, the baseline sPD-L1 level was not an independent prognostic factor for glioma patients $(P=0.516, \mathrm{HR}=2.231,95 \%$ CI: 0.198 25.126). For PFS, patients with sPD-L1 concentrations above $14.35 \mathrm{pg} / \mathrm{ml}$ had worse PFS than those with low sPD-L1 levels (20.4 vs. 26.7 months, $P=0.027$; Figure 3A), but an sPD-L1 concentration $>14.35 \mathrm{pg} / \mathrm{ml}$ was not proven to be an independent prognostic factor for glioma patients in the multivariate analysis. High tumor grade was a poor prognostic factor for PFS both in univariate analysis $(P<0.001)$ (Figure 3D) and in multivariate analysis $(P=0.001, \mathrm{HR}=3.091,95 \% \mathrm{CI}$ : 1.592-6.002) (Table 2). As for other factors, including the change of sPD-L1, IDH-1 mutational status, tumor position, and $\mathrm{Ki}-67$ expression, none of them exhibits a significant correlation with the PFS of glioma patients (Figures 3B, C, E, F).

\section{Radiation Increases SPD-L1 Levels in Glioma Patients}

To explore whether radiation can induce sPD-L1 accumulation, we also measured the sPD-L1 levels in plasma samples of 51 out of 60 patients after RT ( $30 \pm 10 \mathrm{~Gy}, 2 \mathrm{~Gy} / \mathrm{f})$, and found that their 

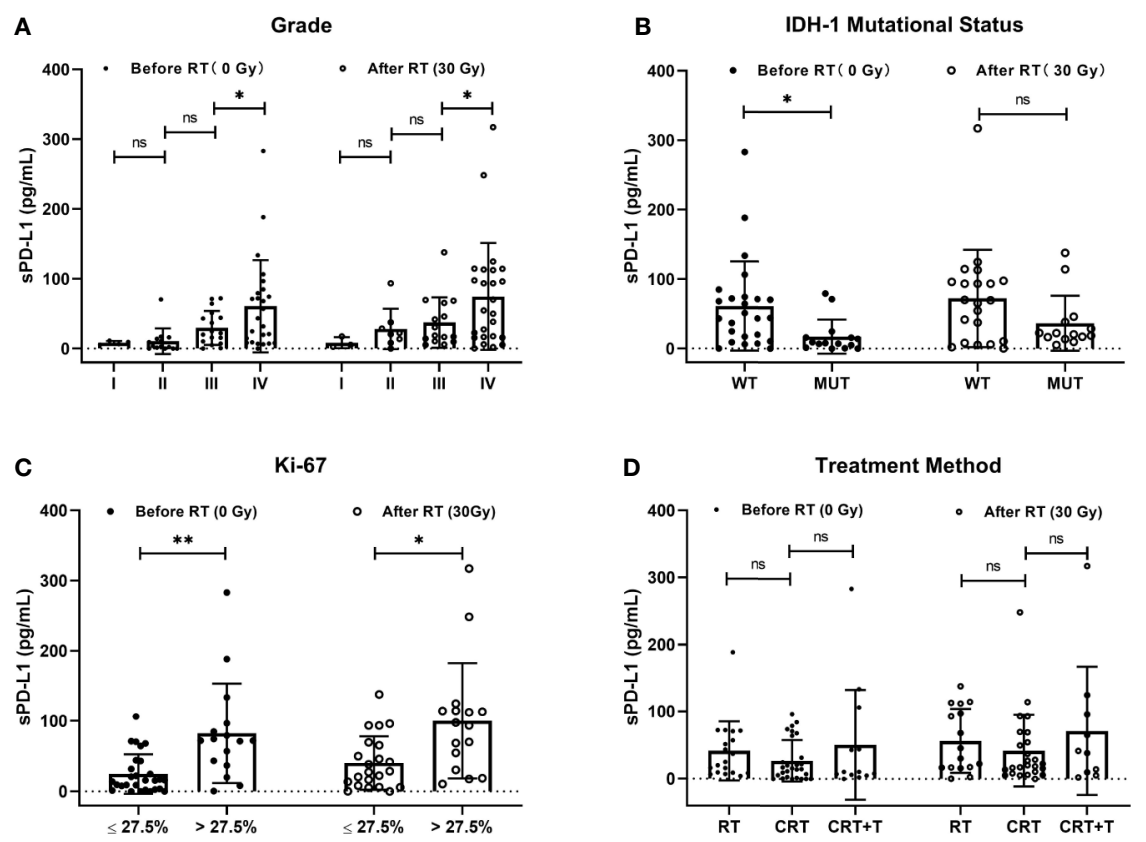

FIGURE 1 | Correlations of soluble PD-L1 (sPD-L1) levels with clinical factors. (A) Grade (I, II, III, and IV), (B) IDH-1 mutational status (mutation and wide type), (C) Ki-67 ( $\leq 27.5 \%$ and $>27.5 \%$ ), and (D) treatment method (Radiotherapy (RT), RT plus chemotherapy (CRT), CRT and beacizumab (CRT+T) before and after RT. ns, non-significant. ${ }^{\star} P<0.05,{ }^{\star \star} P<0.01$.

mean SPD-L1 levels after RT were significantly higher than the baseline sPD-L1 levels before RT $(57.21 \pm 60.95 \mathrm{pg} / \mathrm{ml} v$ s. $36.65 \pm$ $49.77 \mathrm{pg} / \mathrm{ml}, P<0.001$ ) (Figure 4A). In details, sPD-L1 levels were increased in 31 patients and were reduced in 20 patients (Figure 4B).

Next, we undertook further analysis to measure other potential factors that might influence SPD-L1 levels. We first assessed the IDH-I mutational status by pathology analysis. In IDH-1 MUT group, the mean sPD-L1 levels were $17.52 \pm 25.50$ $\mathrm{pg} / \mathrm{ml}$ before RT and $36.60 \pm 39.66 \mathrm{pg} / \mathrm{ml}$ after RT, while they were $66.40 \pm 66.55 \mathrm{pg} / \mathrm{ml}$ before RT and $70.32 \pm 68.96 \mathrm{pg} / \mathrm{ml}$ after RT in the IDH-1 WT group. The baseline sPD-L1 level was significantly higher in the IDH- 1 WT group than in the IDH-1 MUT group $(P=0.016)$; however, there was no statistical significance after RT between the two groups $(P=0.107)$ (Figure 1B). These results showed that SPD-L1 levels tended to increase in these two groups after RT, whereas the fold-change in the IDH-1 MUT group seemed more prominent. Conversely, in the treatment scheme subgroup analysis, the results showed that there were no significant differences in SPD-L1 levels among the RT, CRT, and CRT+T groups either before or after treatment (before treatment: $41.17 \pm 43.85 \mathrm{pg} / \mathrm{ml}, 27.04 \pm 30.96 \mathrm{pg} / \mathrm{ml}$ and $50.73 \pm 81.71 \mathrm{pg} / \mathrm{ml}$, respectively, $P=0.332$; after treatment: $56.69 \pm 47.52 \mathrm{pg} / \mathrm{ml}, 42.18 \pm 53.46 \mathrm{pg} / \mathrm{ml}$, and $71.56 \pm 95.54 \mathrm{pg} /$ $\mathrm{ml}, P=0.432$. Figure 1D). Thus, the addition of chemotherapy and bevacizumab to RT did not further influence the levels of sPD-L1 in this study.

\section{Anti-PD-L1 Antibody Could Reduce the Expression of SPD-L1 in Glioma Murine Model}

To address whether circulating sPD-L1 molecules are directly targeted by an anti-PD-L1 antibody, we performed in vivo studies using the glioma murine model treated with IR (20 Gy), anti-PD-L1 or IR plus anti-PD-L1; we found that there was no difference in baseline sPD-L1 expression levels among the different groups $(1.58 \pm 0.315 \mathrm{pg} / \mathrm{ml}, 1.69 \pm 0.24 \mathrm{pg} / \mathrm{ml}$, and $1.18 \pm 0.51 \mathrm{pg} / \mathrm{ml}$, respectively, $P=0.227$; Figures 5A, B). In line with the clinical data, an increase in the expression of sPD-L1 was observed after IR compared with the expression levels in the nonirradiated control group $(16.68 \pm 11.22 \mathrm{pg} / \mathrm{ml}$ vs. $28.50 \pm$ $11.18 \mathrm{pg} / \mathrm{ml}, P=0.031$, Figure 5B). Notably, the concentration of sPD-L1 could not be detected in either the anti-PD-L1 alone group or the IR plus anti-PD-L1 group (Figure 5B), suggesting that sPD-L1 can be blocked by PD-L1 antibody, which can lead to significant downregulation of sPD-L1.

Since $\mathrm{CD}^{+} \mathrm{T}$ cells is crucial for radiation-induced anti-tumor effects, we sought to examine whether sPD-L1 affects the $\mathrm{CD} 8^{+} \mathrm{T}$ cells activation in the adaptive immune response. We performed the $\mathrm{CD} 8^{+} \mathrm{T}$ cells suppression analysis using sPD-L1-included mice plasma (Figure 5A). The plasma from the mice without anti-PDL1 treatment group exhibited the remarkable $\mathrm{CD}^{+} \mathrm{T}$ cell suppression capacity (Figure $\mathbf{5 C}$ ). The plasma from mice after anti-PD-L1 group (the sPD-L1 concentration is undetectable) didn't show any suppression activity (Figure 5C). These indicate 
A

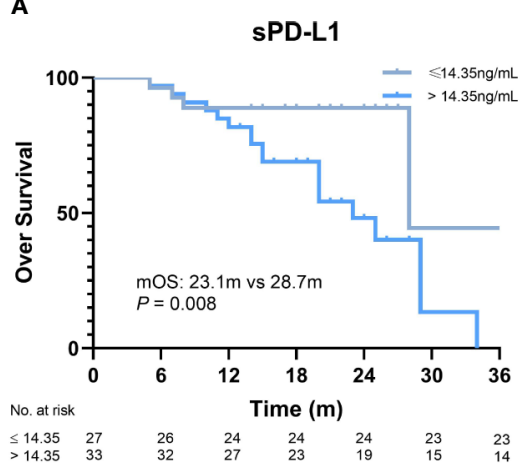

C

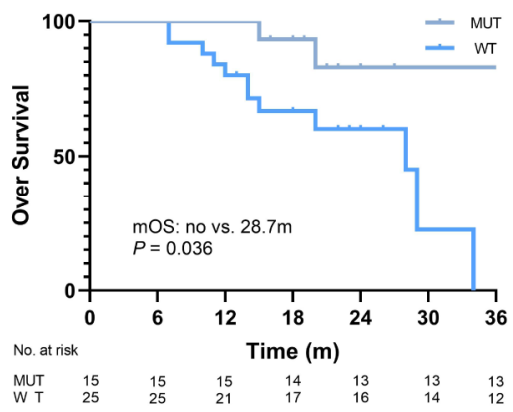

E

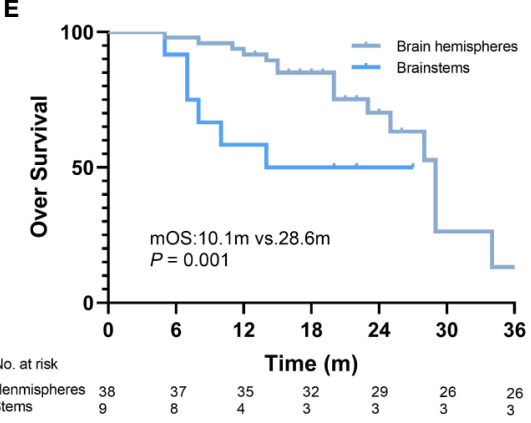

B

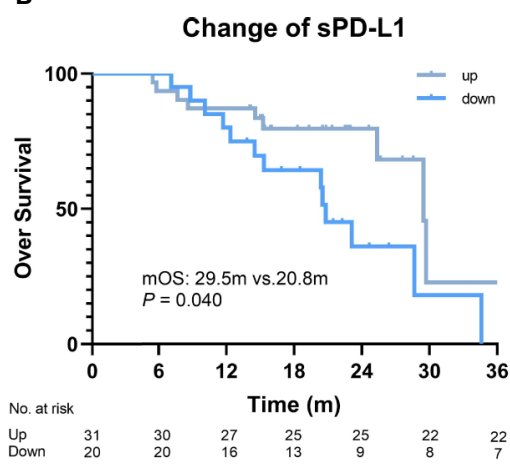

D Grade

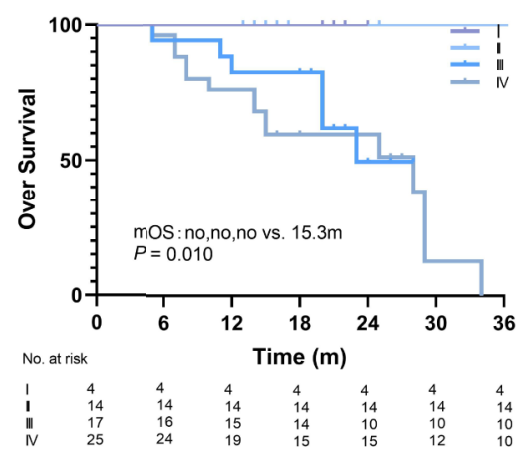

Ki-67

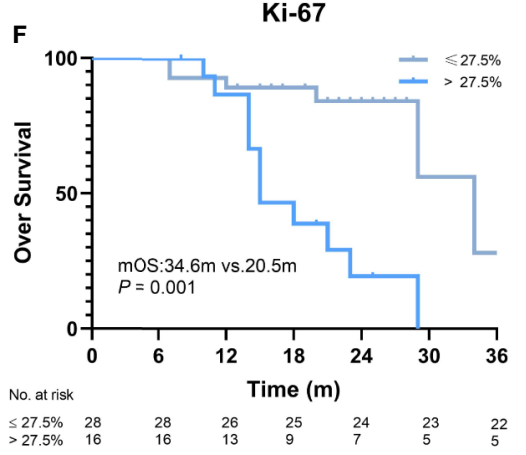

FIGURE 2 | Overall survival (OS) of patients according to different factors. (A) The baseline level of soluble PD-L1 1 (sPD-L1) ( $\leq 14.35 \mathrm{ng} / \mathrm{ml}$ vs. >14.35 ng/ml, $P$ = 0.008). (B) Changes in SPD-L1 after radiotherapy (up vs. down, $P=0.04)$. (C) IDH-1 mutational status (mutation vs. wide type, $P=0.036)$. (D) Grade (I, II, III, vs. IV, $P=0.01$ ). (E) Tumor position (brain hemispheres vs. brainstems, $P=0.001)$. (F) Ki-67 ( $\leq 27.5 \%$ vs. $>27.5 \%, P=0.001)$.

that the sPD-L1 plays an important role in T cell suppression in tumors.

We next sought to validate whether targeting sPD-L1 using anti-PD-L1 antibody can be a potential cancer therapy. We monitored the tumor size in glioma-bearing mice with different treatments. The IR or anti-PD-L1 antibody alone can slow down tumor growth (Figure 5D). Notably, the combination of IR and anti-PD-L1 significantly reduced tumor growth than either monotherapy (anti-PD-L1 vs. IR plus anti-PD-L1: $789.67 \pm 55.86 \mathrm{~mm}^{3}$ vs. $292.16 \pm 102.98 \mathrm{~mm}^{3}$ on day $31, P<$ 0.001; IR vs. IR plus anti-PD-L1 = 697.02 $\pm 12.98 \mathrm{~mm}^{3} v s .292 .16$ $\pm 102.98 \mathrm{~mm}^{3}$ on day $\left.31, P<0.001\right)$ (Figure 5D).

\section{DISCUSSION}

Circulating sPD-L1 in the blood has recently been discovered in various malignancies. However, few studies have reported sPDL1 expression in patients with glioma until now (21). To further explore the existence of sPD-L1 and evaluate the pathological significance of this circulating factor in human cancer, we developed this study for the detection and quantification of sPD-L1 in glioma patients receiving RT. In the present study, by using ELISA formats, we detected that approximately $90 \%$ of glioma patients expressed sPD-L1 in the plasma before RT. Further, we determined that RT could upregulate sPD-L1 
TABLE 2 | Univariable and multivariable analyses of OS and PFS in the patients.

\begin{tabular}{|c|c|c|c|c|c|c|c|c|}
\hline \multirow[t]{3}{*}{ Parameter } & \multicolumn{4}{|c|}{ os } & \multicolumn{4}{|c|}{ PFS } \\
\hline & \multirow{2}{*}{$\frac{\text { Univariate analysis }}{P}$} & \multicolumn{3}{|c|}{ Multivariate analysis } & \multirow{2}{*}{$\frac{\text { Univariate analysis }}{P}$} & \multicolumn{3}{|c|}{ Multivariate analysis } \\
\hline & & $P$ & HR & $95 \% \mathrm{Cl}$ & & $P$ & HR & $95 \% \mathrm{Cl}$ \\
\hline $\begin{array}{l}\text { Gender } \\
\text { (male vs. female) }\end{array}$ & 0.642 & & & & 0.656 & & & \\
\hline $\begin{array}{l}\text { Age } \\
\text { ( } \leq 52 \text { years vs. }>52 \text { years })\end{array}$ & 0.783 & & & & 0.952 & & & \\
\hline $\begin{array}{l}\text { Grade } \\
(I, \text { II, III vs. IV) }\end{array}$ & $0.010^{*}$ & 0.855 & 1.158 & $0.242-5.534$ & $<0.001^{\star}$ & $0.001^{*}$ & 3.091 & $1.592-6.002$ \\
\hline $\begin{array}{l}\text { IDH-1 } \\
\text { (WT vs. MUT) }\end{array}$ & $0.036^{\star}$ & $0.011^{*}$ & 0.029 & $0.002-0.448$ & 0.393 & & & \\
\hline $\begin{array}{l}\text { sPD-L1 } \\
(\leq 14.35 \mathrm{pg} / \mathrm{ml} \text { vs. }>14.35 \mathrm{pg} / \mathrm{ml})\end{array}$ & $0.008^{*}$ & 0.516 & 2.231 & $0.198-25.126$ & $0.027^{\star}$ & 0.617 & 0.737 & $0.223-2.435$ \\
\hline $\begin{array}{l}\text { Ki-67 } \\
(\leq 27.5 \% \text { vs. }>27.5 \%)\end{array}$ & $0.001^{*}$ & 0.846 & 0.824 & $0.117-5.818$ & 0.066 & 0.635 & 1.278 & $0.464-3.524$ \\
\hline $\begin{array}{l}\text { Position } \\
\text { (brain hemispheres vs. brainstem) }\end{array}$ & $0.001^{*}$ & $0.002^{*}$ & 26.302 & $3.239-213.550$ & 0.876 & & & \\
\hline $\begin{array}{l}\text { Treatment } \\
\text { (RT vs. RT + chemotherapy } \pm \text { target }\end{array}$ & 0.708 & & & & 0.715 & & & \\
\hline Change in sPD-L1 (up vs. down) & $0.040^{*}$ & $0.003^{*}$ & 0.019 & $0.001-0.268$ & 0.770 & & & \\
\hline
\end{tabular}

Factors with $P \leq 0.1$ in univariate analysis can be included in the multivariate analysis.

OS, overall survival; PFS, progression-free survival; $\mathrm{HR}$, hazard ratio; $\mathrm{Cl}$, confidence interval. ${ }^{*} P \leq 0.05$.

levels compared with baseline levels. In addition, the high baseline level of sPD-L1, decreased level of sPD-L1 after RT and some other clinical factors, such as IDH-1 WT tumors, GBMs, tumors located in the brainstem and tumors with a Ki-67 expression rate $>27.5 \%$, were demonstrated to be related to poor prognosis in glioma patients. Using the glioma murine model, our data showed that anti-PD-L1 antibody combined with radiation can be an effective therapy method.

The PD-L1/PD-L1 axis is associated with the tumor microenvironment as a regulator of inhibitory signals, and its expression could be a candidate biomarker for patient selection for anti-PD-L1/PD-L1 monoclonal therapy. Aberrant PD-L1 expression has already been reported to occur in glioma tumor tissues based on IHC $(8,23)$. Considering that the sPD-L1 level may be associated with the tumor burden and the aggressive biological activities of tumors, we investigated whether there were associations between the baseline level of sPD-L1 and the tumor grade or Ki-67 expression, and finally demonstrated that the baseline level of sPDL1 was significantly elevated in patients with advanced brain tumors or in patients with $\mathrm{Ki}-67>27.5 \%$. Ki-67 is one of the most widely used markers for proliferation in clinical practice and has been validated as a marker in the initial phase of adult neurogenesis (24). Although the mechanism remains unclear, we speculated that most sPD-L1 may be shed from the surface of cells in tumors by the cleavage of membrane-bound proteins and is found in free form in the plasma. In addition, circulating sPD-L1 could lead to immune tolerance; consequently, neoplastic cells would have no limits to proliferation. Therefore, sPD-L1 may be considered to exist from the early stage of glioma progression. In recent years, distinct molecular classes of gliomas have been identified. It is well established that IDH-1 MUT and IDH-1 WT gliomas have distinct tumor behavior driven by different oncogenic signals and respond differently to current treatment paradigms. Notably, by comparing the immune responses between IDH-1 MUT and IDH1 WT patients, some studies have identified a marked reduction in the expression of immune-related genes, including the CD274 (PDL1 coding gene) gene, in IDH-1 MUT gliomas (25-27). The results were in line with ours, and downregulated SPD-L1 levels tended to occur in glioma patients with IDH-1 MUT tumors. Collectively, these findings may suggest that the immunological tumor microenvironment may differ according to the IDH mutational status of gliomas.

In addition to the association with some clinical factors, sPD-L1 levels might predict the survival outcomes in cancer patients; however, its prognostic relevance was contradictory in different cancers. In gastric adenocarcinoma, elevated levels of sPD-L1 were associated with a favorable prognosis $(65.6 \%$ vs. $44.7 \%, P=0.028)$ (28). Inversely, studies in natural killer/T-cell lymphoma (NKTCL), aggressive renal cell carcinoma (RCC), NSCLC, large B-cell lymphoma, HCC, and nasopharynx cancer (NPC) demonstrated that patients with high concentrations of sPD-L1 exhibited markedly worse survival than patients with lower concentrations $(18-20,29,30)$. In the current study, we observed that high baseline levels of sPD-L1 (>14.35 pg/ml) in glioma patients were correlated not only with poorer OS (23.1 vs. 28.7 months, $P=0.008)$ but also with poorer PFS (20.4 vs. 26.7 months, $P=0.027)$ in univariate analysis. The biological reason why sPD-L1 is more strongly associated with survival outcomes has to be further elucidated. It is very possible that as SPD-L1 spreads throughout the body via the blood and lymphatic circulation, it exerts a widespread inhibitory effect on $\mathrm{T}$ cells by interacting with cell surface receptors such as membrane-bound PD-1 (31-33). And this hypothesis was verified by $\mathrm{T}$ cell suppression assay in this study. In addition, we found that sPD-L1 molecules might represent a direct target of therapeutic PDL1 antibodies. The described functions might work as mechanisms of escape from immune surveillance and/or result in an impairment 
A

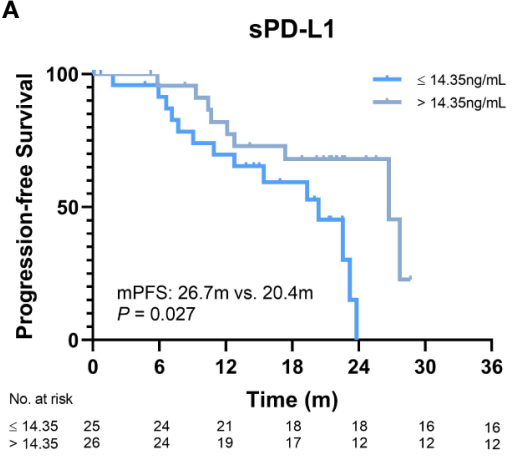

C

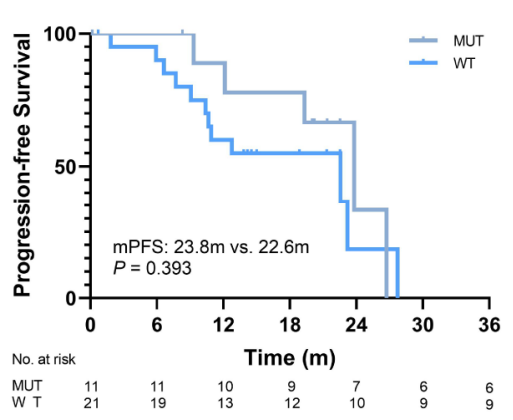

E

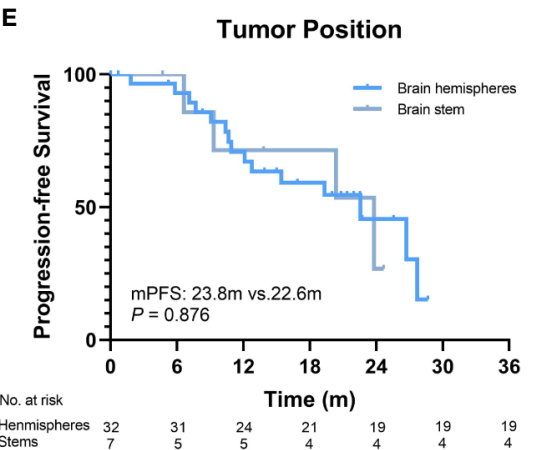

B

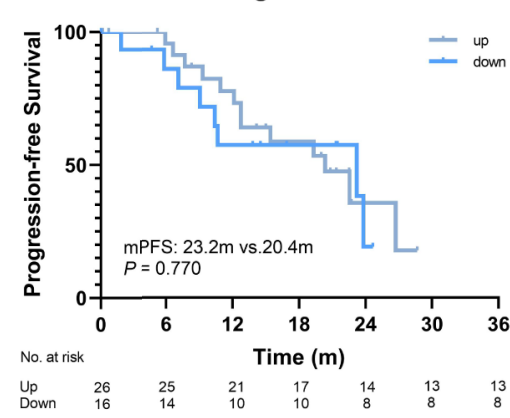

D

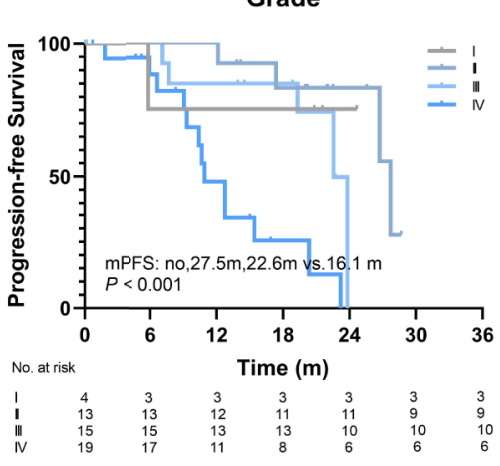

$\mathbf{F}$

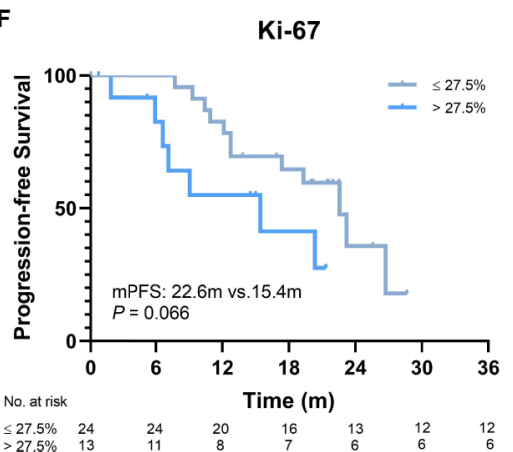

FIGURE 3 | Progression-free survival (PFS) of patients according to different factors. (A) The baseline level of soluble programmed death-ligand 1 (sPD-L1) ( $\leq 14.35$ $\mathrm{ng} / \mathrm{ml}$ vs. $>14.35 \mathrm{ng} / \mathrm{ml}, P=0.027$ ). (B) Changes in SPD-L1 after radiotherapy (up vs. down, $P=0.770$ ). (C) IDH-1 mutational status (mutation vs. wide type, $P=$ 0.393). (D) Grade (I, II, III vs. IV, $P<0.001$ ). (E) Tumor position (brain hemispheres vs. brainstems, $P=0.876)$. (F) Ki-67 ( $\leq 27.5 \%$ vs. $>27.5 \%, P=0.066)$.

of anti-PD-1/PD-L1-directed antibody therapy and thus translate into prognostic and/or predictive factors in cancer patients. Altogether, the quantification of circulating sPD-L1 may also be of use as a predictive marker of anti-PD-1 treatment outcome, helping clinicians select patients for PD-1/PD-L1-based therapy strategies. Thus, further studies with a large number of patients are required to clarify our findings.

Next, we attempted to uncover the dynamics of circulating sPD-L1 levels in glioma patients undergoing RT. It is known that RT complicates the interpretation of the immune landscape in patients. The ultimate goal of the combination of immunotherapy and RT is to achieve a long-lasting, therapyinduced immune response at all sites of disease, and the assessment of the dynamic changes in the immune system at the patient level is essential. Our group analyzed the changes in the sPD-L1 level before and following RT and found that RT significantly increased SPD-L1 expression in both patients with glioma and glioma murine model. Similarly, Hyun et al. reported that RT significantly increased sPD-L1 expression in patients with HCC (34). However, most investigations have focused only on the PD-L1 level at baseline, and data on changes in PD-L1 expression after RT are still extremely limited. 

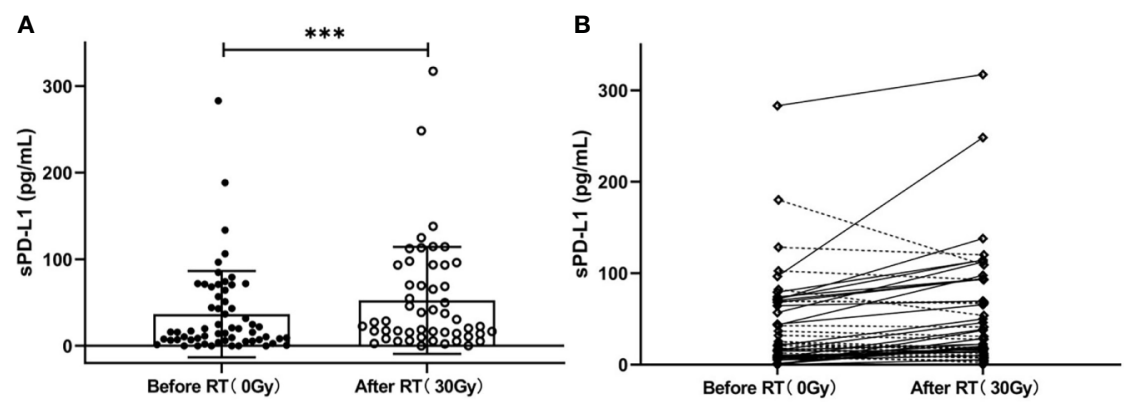

FIGURE 4 | Changes in the soluble PD-L1 (sPD-L1) levels after radiotherapy (RT). (A) Overall change in sPD-L1 levels in patients (before RT vs. after RT: $36.65 \pm$ $49.77 \mathrm{pg} / \mathrm{ml}$ vs. $57.21 \pm 60.95 \mathrm{pg} / \mathrm{ml}, P<0.001)$. (B) Individual change in SPD-L1 levels (increased in 31 patients and reduced in 20 patients). ${ }^{\star \star \star} P<0.001$. Data are presented as mean \pm SD.

A



C

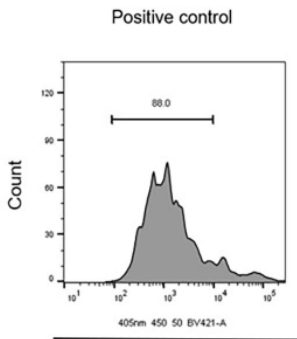

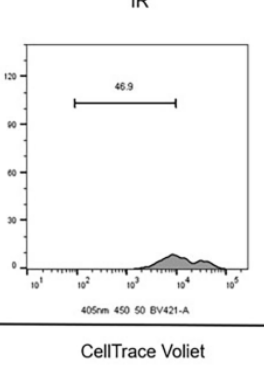

IR + Anti-PD-L1

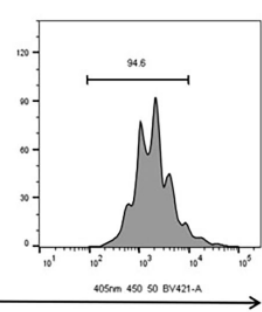

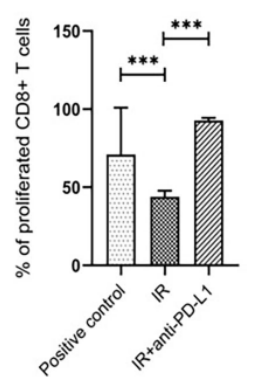

B

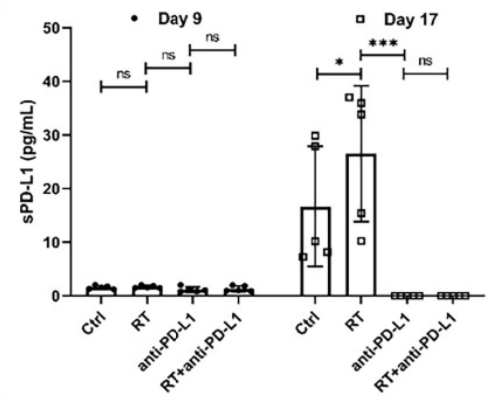

D

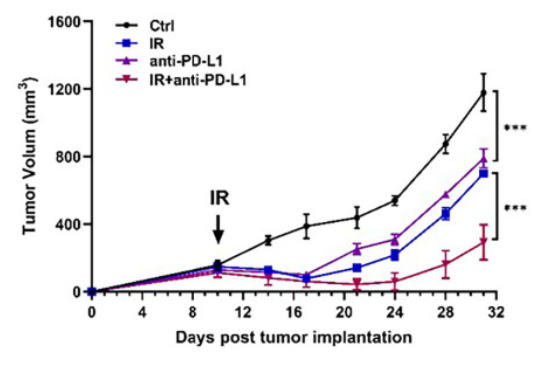

FIGURE 5 | Anti-PD-L1 antibody could reduce the expression of SPD-L1 in glioma murine model. The glioma murine models were divided into ionizing radiation (IR) (20 Gy), anti-PD-L1, IR plus anti-PD-L1, and control groups $(n=5)$. (A) The scheme of in vivo experiments using glioma murine model. (B) The soluble PD-L1 (sPDL1) were measured before and after IR, respectively, there was no difference in baseline expression levels in different groups; however, IR can upregulate the expression of SPD-L1 and anti-PD-L1 can effectively reduce the expression of SPD-L1. (C) Naive CD8+ T cells were cocultured with the plasma of mice after IR and subjected to suppression assay. Ratio indicates proliferated CD8+ T cells. It showed that suppression on T-cell proliferation can be enhanced upon sPD-L1 incubation in vitro. (D) Treated tumor was measured every 3-4 days for 21 days starting from the day of IR. The combination of IR and anti-PD-L1 could reduce tumor growth than either monotherapy. ns, nonsignificant. ${ }^{\star} \mathrm{P}<0.05$; ${ }^{\star \star \star} \mathrm{P}<0.001$. Each experiment was repeated three times. Data are presented as mean $\pm \mathrm{SD}$.

In the sub-analysis of this study, we also noticed that SPD-L1 levels were increased significantly in IDH-1 MUT patients compared with IDH-1 WT patients. To the best of our knowledge, this is the first prospective study to evaluate the SPDL1 levels following RT in glioma patients with different IDH mutational statuses. Our finding can be explained by the fact that IDH-1 mutation could apparently improve the sensitivity of tumors to RT, and then cells in tumors killed by RT could release an abundance of sPD-L1 into the blood. However, we also found that the addition of chemotherapy or/and bevacizumab to RT did not further upregulate the increase in sPD-L1 compared with RT only. We suspect that RT as a local therapy provides sufficient damage to the tumor target, and there was probably no room to further improve treatment efficacy with the addition of chemotherapy 
and/or bevacizumab beyond that of RT in the primary tumor. Taken together, the sPD-L1 level increased after RT, suggesting that RT combined with immune checkpoint inhibitors might be better than RT alone for glioma patients, especially for patients with IDH1 MUT. Further well-designed studies are needed to clarify the optimal RT scheme, dose, and time for combination.

Regarding the limitations of this study, first, this study had a limited sample size. Second, the only RT dose used was $30 \pm 10$ Gy; hence, we could not determine whether the sPD-L1 level will change after a larger dose of RT. Third, some patients underwent partial excision, and others underwent only biopsy, which might affect the sPD-L1 level.

\section{CONCLUSION}

In conclusion, this study reported that SPD-L1 can be assayed in the plasma of glioma patients. This finding may mean that compensation for the potential sequestration of antibodies needs to be considered in the optimization of PD-L1 blockade therapies. Not all administered anti-PD-L1 immunotherapeutic antibodies may reach the surface of tumor cells, with a potentially appreciable proportion being sequestered by sPDL1 within the circulation. In addition, the baseline level of sPDL1 might be a potential marker to predict the outcome in glioma patients, which would truly be remarkable, because predictive biomarkers that discriminate responders from non-responders at therapy initiation are scarce. Finally, the elevated level of sPD-L1 after RT suggests that the combination of RT with immune checkpoint inhibitors may be a promising therapeutic strategy in gliomas, especially for patients with IDH-1 MUT gliomas.

\section{DATA AVAILABILITY STATEMENT}

The original contributions presented in the study are included in the article/supplementary materials. Further inquiries can be directed to the corresponding authors.

\section{REFERENCES}

1. Ostrom QT, Gittleman H, Truitt G, Boscia A, Kruchko C, Barnholtz-Sloan JS. CBTRUS Statistical Report: Primary Brain and Other Central Nervous System Tumors Diagnosed in the United States in 2011-2015. Neuro Oncol (2018) 20 (suppl_4):iv1-86. doi: 10.1093/neuonc/noy131

2. Lim M, Xia Y, Bettegowda C, Weller M. Current state of immunotherapy for glioblastoma. Nat Rev Clin Oncol (2018) 15(7):422-42. doi: 10.1038/s41571018-0003-5

3. Overman MJ, Lonardi S, Wong KYM, Lenz H-J, Gelsomino F, Aglietta $\mathrm{M}$, et al. Durable clinical benefit with nivolumab plus ipilimumab in DNA mismatch repair-deficient/microsatellite instability-high metastatic colorectal cancer. J Clin Oncol (2018) 36(8):773-9. doi: 10.1200/JCO.2017.76.9901

4. Motzer RJ, Tannir NM, McDermott DF, Arén Frontera O, Melichar B, Choueiri TK, et al. Nivolumab plus ipilimumab versus sunitinib in advanced renal-cell carcinoma. New Engl J Med (2018) 378(14):1277-90. doi: 10.1056/NEJMoa1712126

\section{ETHICS STATEMENT}

The studies involving human participants were reviewed and approved by The Ethics Committee of the Shandong Cancer Hospital. The patients/participants provided their written informed consent to participate in this study. The animal study was reviewed and approved by The Ethics Committee of the Shandong Cancer Hospital.

\section{AUTHOR CONTRIBUTIONS}

$\mathrm{X}-\mathrm{CD}$ was a major contributor in writing the manuscript. L-LW and Y-FZ analyzed and interpreted the patient and animal data. $\mathrm{X}-\mathrm{CD}$ and JY did the experiments in the manuscript. J-MY, HL, RRW and $\mathrm{MH}$ designed the work and provided the financial support. S-LN and Y-DL revised the manuscript. All authors contributed to the article and approved the submitted version.

\section{FUNDING}

This work was supported by National Key Research and Development Program of China (Grant No. 2018YFE0114100), Key Research and Development Program of Shandong province, China (Grant No. 2019GGX101057), Science Technology Program of Jinan (Grant No. 201805051) and The Innovation Project of Shandong Academy of Medical Sciences (2019-04), and the Academic Promotion Program of Shandong First Medical University (Grant No. 2019ZL002).

\section{ACKNOWLEDGMENTS}

We thank the patients who participated in this study, their families, and the staff members at the study sites who cared for them. This manuscript has been released as a pre-print at Research Square (35).

5. Ansell SM, Lesokhin AM, Borrello I, Halwani A, Scott EC, Gutierrez M, et al. PD1 blockade with nivolumab in relapsed or refractory Hodgkin's lymphoma. New Engl J Med (2015) 372(4):311-9. doi: 10.1056/NEJMoa1411087

6. Wang L, Gao Y, Zhang G, Li D, Wang Z, Zhang J, et al. Enhancing KDM5A and TLR activity improves the response to immune checkpoint blockade. Sci Trans Med (2020) 12(560):eaax2282. doi: 10.1126/scitranslmed.aax2282

7. Maxwell R, Jackson CM, Lim M. Clinical Trials Investigating Immune Checkpoint Blockade in Glioblastoma. Curr Treat Options Oncol (2017) 18 (8):51. doi: 10.1007/s11864-017-0492-y

8. Xue S, Hu M, Iyer V, Yu J. Blocking the PD-1/PD-L1 pathway in glioma: a potential new treatment strategy. J Hematol Oncol (2017) 10(1):81. doi: 10.1186/s13045-017-0455-6

9. Filley AC, Henriquez M, Dey M. Recurrent glioma clinical trial, CheckMate143: the game is not over yet. Oncotarget (2017) 8(53):91779. doi: 10.18632/ oncotarget. 21586

10. Rajani KR, Carlstrom LP, Parney IF, Johnson AJ, Warrington AE, Burns TC. Harnessing Radiation Biology to Augment Immunotherapy for Glioblastoma. Front Oncol (2019) 8:656. doi: 10.3389/fonc.2018.00656 
11. Nesseler JP, Schaue D, McBride WH, Lee M-H, Kaprealian T, Niclou SP, et al. Irradiation to Improve the Response to Immunotherapeutic Agents in Glioblastomas. Adv Radiat Oncol (2019) 4(2):268-82. doi: 10.1016/ j.adro.2018.11.005

12. Sahebjam S, Sharabi A, Lim M, Kesarwani P, Chinnaiyan P. Immunotherapy and radiation in glioblastoma. J Neuro Oncol (2017) 134(3):531-9. doi: 10.1007/s11060-017-2413-0

13. Shen X, Zhao B. Efficacy of PD-1 or PD-L1 inhibitors and PD-L1 expression status in cancer: meta-analysis. BMJ (2018) 362:k3529. doi: 10.1136/bmj.k3529

14. Horn L, Spigel DR, Vokes EE, Holgado E, Ready N, Steins M, et al. Nivolumab Versus Docetaxel in Previously Treated Patients With Advanced Non-SmallCell Lung Cancer: Two-Year Outcomes From Two Randomized, Open-Label, Phase III Trials (CheckMate 017 and CheckMate 057). J Clin Oncol Off J Am Soc Clin Oncol (2017) 35(35):3924-33. doi: 10.1200/JCO.2017.74.3062

15. Balermpas P, Rödel F, Rödel C, Krause M, Linge A, Lohaus F, et al. CD8+ tumour-infiltrating lymphocytes in relation to HPV status and clinical outcome in patients with head and neck cancer after postoperative chemoradiotherapy: A multicentre study of the German cancer consortium radiation oncology group (DKTK-ROG). Int J Cancer (2016) 138(1):171-81. doi: $10.1002 /$ ijc. 29683

16. Afanasiev OK, Yelistratova L, Miller N, Nagase K, Paulson K, Iyer JG, et al. Merkel polyomavirus-specific $\mathrm{T}$ cells fluctuate with merkel cell carcinoma burden and express therapeutically targetable PD-1 and Tim-3 exhaustion markers. Clin Cancer Res Off J Am Assoc Cancer Res (2013) 19(19):5351-60. doi: 10.1158/1078-0432.CCR-13-0035

17. Grassberger C, Ellsworth SG, Wilks MQ, Keane FK, Loeffler JS. Assessing the interactions between radiotherapy and antitumour immunity. Nat Rev Clin Oncol (2019) 16(12):729-45. doi: 10.1038/s41571-019-0238-9

18. Zhang J, Gao J, Li Y, Nie J, Dai L, Hu W, et al. Circulating PD-L 1 in NSCLC patients and the correlation between the level of PD-L 1 expression and the clinical characteristics. Thoracic Cancer (2015) 6(4):534-8. doi: 10.1111/1759-7714.12247

19. Rossille D, Gressier M, Damotte D, Maucort-Boulch D, Pangault C, Semana $\mathrm{G}$, et al. High level of soluble programmed cell death ligand 1 in blood impacts overall survival in aggressive diffuse large B-Cell lymphoma: results from a French multicenter clinical trial. Leukemia (2014) 28(12):2367-75. doi: 10.1038/leu.2014.137

20. Finkelmeier F, Canli Ö, Tal A, Pleli T, Trojan J, Schmidt M, et al. High levels of the soluble programmed death-ligand (sPD-L1) identify hepatocellular carcinoma patients with a poor prognosis. Eur J Cancer (2016) 59:152-9. doi: 10.1016/j.ejca.2016.03.002

21. Liu S, Zhu Y, Zhang C, Meng X, Sun B, Zhang G, et al. The Clinical Significance of Soluble Programmed Cell Death-Ligand 1 (sPD-L1) in Patients With Gliomas. Front Oncol (2020) 10:9. doi: 10.3389/fonc.2020.00009

22. Ugurel S, Schadendorf D, Horny K, Sucker A, Schramm S, Utikal J, et al. Elevated baseline serum PD-1 or PD-L1 predicts poor outcome of PD-1 inhibition therapy in metastatic melanoma. Ann Oncol (2020) 31(1):144-52. doi: 10.1016/j.annonc.2019.09.005

23. Xue S, Hu M, Li P, Ma J, Xie L, Teng F, et al. Relationship between expression of PD-L1 and tumor angiogenesis, proliferation, and invasion in glioma. Oncotarget (2017) 8(30):49702-12. doi: 10.18632/oncotarget.17922

24. Kee N, Sivalingam S, Boonstra R, Wojtowicz JM. The utility of Ki-67 and BrdU as proliferative markers of adult neurogenesis. J Neurosci Methods (2002) 115(1):97-105. doi: 10.1016/S0165-0270(02)00007-9
25. Mu L, Long Y, Yang C, Jin L, Tao H, Ge H, et al. The IDH1 Mutation-Induced Oncometabolite, 2-Hydroxyglutarate, May Affect DNA Methylation and Expression of PD-L1 in Gliomas. Front Mol Neurosci (2018) 11:82. doi: 10.3389/fnmol.2018.00082

26. Berghoff AS, Kiesel B, Widhalm G, Wilhelm D, Rajky O, Kurscheid S, et al. Correlation of immune phenotype with IDH mutation in diffuse glioma. Neuro-oncology (2017) 19(11):1460-8. doi: 10.1093/neuonc/nox054

27. Choi BD, Curry WT. IDH mutational status and the immune system in gliomas: a tale of two tumors? Trans Cancer Res (2017) 6(Suppl 7):S1253-s6. doi: 10.21037/tcr.2017.09.37

28. Zheng Z, Bu Z, Liu X, Zhang L, Li Z, Wu A, et al. Level of circulating PD-L1 expression in patients with advanced gastric cancer and its clinical implications. Chin J Cancer Res (2014) 26(1):104-11. doi: 10.3978/j.issn.1000-9604.2014.02.08

29. Yang J, Hu M, Bai X, Ding X, Xie L, Ma J, et al. Plasma levels of soluble programmed death ligand 1 (sPD-L1) in WHO II/III nasopharyngeal carcinoma (NPC): A preliminary study. Medicine (2019) 98(39):e17231. doi: 10.1097/MD.0000000000017231

30. Frigola X, Inman BA, Lohse CM, Krco CJ, Cheville JC, Thompson RH, et al. Identification of a soluble form of B7-H1 that retains immunosuppressive activity and is associated with aggressive renal cell carcinoma. Clin Cancer Res Off J Am Assoc Cancer Res (2011) 17(7):1915-23. doi: 10.1158/1078-0432.CCR-10-0250

31. Ludwig S, Floros T, Theodoraki MN, Hong CS, Jackson EK, Lang S, et al. Suppression of Lymphocyte Functions by Plasma Exosomes Correlates with Disease Activity in Patients with Head and Neck Cancer. Clin Cancer Res OffJ Am Assoc Cancer Res (2017) 23(16):4843-54. doi: 10.1158/1078-0432.CCR16-2819

32. Theodoraki MN, Yerneni SS, Hoffmann TK, Gooding WE, Whiteside TL. Clinical Significance of PD-L1(+) Exosomes in Plasma of Head and Neck Cancer Patients. Clin Cancer Res Off J Am Assoc Cancer Res (2018) 24(4):896905. doi: 10.1158/1078-0432.CCR-17-2664

33. Frigola X, Inman BA, Krco CJ, Liu X, Harrington SM, Bulur PA, et al. Soluble B7-H1: differences in production between dendritic cells and T cells. Immunol Lett (2012) 142(1-2):78-82. doi: 10.1016/j.imlet.2011.11.001

34. Kim HJ, Park S, Kim KJ, Seong J. Clinical significance of soluble programmed cell death ligand-1 (sPD-L1) in hepatocellular carcinoma patients treated with radiotherapy. Radiother Oncol J Eur Soc Ther Radiol Oncol (2018) 129(1):1305. doi: 10.1016/j.radonc.2017.11.027

35. Xing-chen D, Liang-liang W, Yu-fang Z, Jia Y, Jin-ming Y, Man H. The change of Soluble Programmed Cell Death-Ligand 1 (sPD-L1) in glioma patients receiving radiotherapy and its impact on clinical outcome. Research Square. PREPRINT (Version 1) (2020). doi: 10.21203/rs.3.rs-38703/v1

Conflict of Interest: The authors declare that the research was conducted in the absence of any commercial or financial relationships that could be construed as a potential conflict of interest.

Copyright (C) 2020 Ding, Wang, Zhu, Li, Nie, Yang, Liang, Weichselbaum, Yu and Hu. This is an open-access article distributed under the terms of the Creative Commons Attribution License (CC BY). The use, distribution or reproduction in other forums is permitted, provided the original author(s) and the copyright owner(s) are credited and that the original publication in this journal is cited, in accordance with accepted academic practice. No use, distribution or reproduction is permitted which does not comply with these terms. 